transfusion reaction. 25/30 adverse events occurred after a pRBCs transfusion; 5/30 after WB transfusion. The portion of adverse reactions was (37/129) with pRBCs and (7/36) after WB. 23/44 (52\%) reactions occurred in dogs receiving single transfusions; $17 / 44$ (38\%) occurred after multiple transfusions. Of the 43 dogs receiving multiple transfusions, 10 (23\%) had repeated reactions.

\section{STATEMENT (CONCLUSIONS)}

Immune-mediated disease was the commonest cause of transfusion-dependent anaemia. Treatment with corticosteroids did not decrease the incidence of transfusion reactions. Performance of more than one transfusion was not associated with an increased risk of adverse reactions.

\section{The influence of prior corticosteroid administration on diagnostic imaging findings in dogs with Immune-Mediated Haemolytic Anaemia}

\section{Joshua Hardwick ${ }^{1}$, Jennifer Reeve ${ }^{2}$}

1 University of Bristol, Bristol, United Kingdom

2 Langford Veterinary Services, Bristol, United Kingdom

\section{OBJECTIVES}

To evaluate whether prior corticosteroid administration influences imaging findings in dogs with immunemediated haemolytic anaemia (IMHA).

\section{METHODS}

Retrospective review of case records of a referral population of dogs with IMHA, for whom medical histories and thoracic and/or abdominal imaging studies were available.

Cases were classified based upon presence $(\mathrm{S}+)$ or absence ( $\mathrm{S}-$ ) of corticosteroid administration prior to referral. Thoracic and abdominal imaging findings were categorized into (a) clinically significant findings altering case management or follow-up, including potential triggers; (b) incidental, clinically insignificant; (c) attributable to IMHA; (d) normal. For statistical analysis, significant imaging findings (a) versus non-significant imaging findings ( $b, c$ and $d$ ) were compared in $\mathrm{S}+$ and $\mathrm{S}-$ dogs, using $\chi^{2}$ test to determine whether prior corticosteroids significantly influenced imaging findings.

\section{RESULTS}

55 dogs met the inclusion criteria, comprising $23 \mathrm{~S}+$ and $32 \mathrm{~S}-.46$ had thoracic and abdominal imaging. 2 and 7 dogs had only thoracic or abdominal imaging, respectively. The 48 thoracic studies comprised 25 computedtomographic and 23 plain radiographic. The 53 abdominal studies comprised 24 computed-tomographic and 29 ultrasonographic.

Thoracic imaging findings were clinically significant in 6/19 S+dogs and 6/29 S-dogs. Abdominal imaging findings were clinically significant in 13/22 S+ dogs and 19/31 S- dogs. Incidence of clinically significant thoracic or abdominal imaging findings did not differ between $S+$ and $S-\operatorname{dogs}(p<0.05)$.

\section{STATEMENT (CONCLUSIONS)}

In this population, prior corticosteroid administration did not significantly influence imaging findings. Imaging for underlying disease in IMHA is therefore considered worthwhile, even if corticosteroids have been administered.

\section{Presenting signs and clinical outcome in dogs with metaphyseal osteopathy, 32 cases (2009-2018)}

\author{
Alison Robertson ${ }^{1}$, Sophie Adamantos ${ }^{2}$, \\ Victoria Black ${ }^{1}$, Katherine Clarke ${ }^{3}$, Ian Faux ${ }^{4}$
}

University of Bristol, Bristol, United Kingdom

Langford Vets, Bristol, United Kingdom

Davies Veterinary Specialists, Hitchin, United Kingdom

Royal (Dick) School of Veterinary Studies, Edinburgh,

United Kingdom

\section{OBJECTIVES}

To describe presentation, treatment, and clinical outcome of dogs with metaphyseal osteopathy.

\section{METHODS}

Multi-centre retrospective review of medical records from January 2009 to September 2018 at three referral 